\title{
Caractérisation thermomécanique et endommagement de membranes sous pression de gaz
}

\author{
Patrice Jouinot ${ }^{\mathrm{a}}$ et Vladimir Gantchenko \\ ISMEP-LISMMA, 3 rue Fernand Hainaut, 93407 Saint-Ouen, France
}

Reçu le 23 mars 2007, accepté le 11 décembre 2008

\begin{abstract}
Résumé - Les calculs numériques sont de plus en plus performants pour estimer la durée de vie des structures soumises à des sollicitations thermomécaniques. Mais les durées de vie estimées ne sont correctes que si les paramètres nécessaires aux calculs ont été correctement identifiés et mesurés et si toutes les origines de l'endommagement ont bien été considérées. L'essai de disques sous pression de gaz est une technique expérimentale qui permet la caractérisation thermomécanique des matériaux en feuilles dans des conditions très variées : charge croissante (essais de rupture en traction biaxiale à des vitesses de déformation comprises entre $10^{-6}$ et $10^{0} \mathrm{~s}^{-1}$ ), charge constante à haute température (fluage sous contrainte élevée), charge cyclique (fatigue mécanique) ; la température peut être choisie entre 20 et $900{ }^{\circ} \mathrm{C}$. La technique révèle rapidement l'endommagement du matériau grâce à la détection sensible de fuites au travers de la membrane par un spectromètre de masses. Nous avons développé une méthode de calcul pour déterminer les propriétés mécaniques rationnelles du matériau. Nous montrons la qualité des résultats obtenus avec cette méthode pour la caractérisation thermomécanique des matériaux en feuilles et sa sensibilité à des paramètres secondaires. La méthode analytique de calcul pourra être optimisée de façon à modéliser au mieux le comportement thermomécanique des matériaux sollicités dans des gammes très larges de vitesse et de température et soumis à différents environnements comme de l'hydrogène gazeux par exemple.
\end{abstract}

Mots clés : Propriétés thermomécaniques / lois de comportement thermomécanique / rupture monotone / fluage / fatigue oligocyclique / essais de disques / alliage de cuivre

\begin{abstract}
Mechanical behaviour and damage of thin sheet under gaseous pressure. Numerical calculations become a more and more efficient way to estimate the lifetime of structures under thermomechanical loading. But these life estimations cannot be reliable if the needed parameters have not been correctly identified and measured and if not all the damage causes have been considered. The disk testing under gas pressure is an experimental technique used for the mechanical characterization of thin sheets with various conditions: monotonous loading (biaxial rupture tests at strain rates from $10^{-6}$ to $10^{0} \mathrm{~s}^{-1}$ ), constant loading under high stresses (sustained load) at elevated temperature (creep tests), cyclic loading (mechanical fatigue tests); the temperature may be chosen between 20 and $900{ }^{\circ} \mathrm{C}$. The disk testing quickly reveals the damage when crossing cracks through the membrane create leakages detected by a mass spectrometer. Besides, we have developed a calculation method in order to measure the true mechanical properties of the pressurized disk. We evidence the quality of the results obtained with this method in the thermomechanical characterization of thin sheets and its sensitivity to secondary parameters. The analytical method of calculation will be optimized in order to obtain the best modelling of thermomechanical behaviour of materials loaded at temperature and strain rates within very large ranges and subject to different environments as gaseous hydrogen.
\end{abstract}

Key words: Thermomechanical properties / behaviour modelling / monotonic rupture / creep / low cycle fatigue / disk pressure testing / copper alloy

a Auteur pour correspondance : jouinot@supmeca.fr 


\section{Introduction}

Les calculs par éléments-finis en petites ou grandes déformations sont de plus en plus performants pour dimensionner les structures mécaniques et prévoir leur endommagement; les codes de calculs utilisent des lois qui modélisent le comportement thermomécanique des matériaux.

Pour que les codes de calcul donnent des résultats réalistes, les lois de comportement thermomécanique doivent être bien adaptées à la modélisation des structures en service et leurs paramètres doivent être correctement identifiés; le choix des techniques expérimentales est donc déterminant pour obtenir une caractérisation thermomécanique réaliste.

Par exemple, l'endommagement des parois fines d'échangeurs thermiques, de moteurs de fusée ou de compensateurs de dilatation est mieux corrélé au comportement des matériaux en feuilles qu'à celui d'éprouvettes massives.

Depuis une quarantaine d'années, l'essai de fragilisation de disques sous pression de gaz est utilisé pour étudier la sensibilité à l'hydrogène gazeux des métaux; la sensibilité et la reproductibilité de cette technique expérimentale sont bien meilleures que celles obtenues avec d'autres essais de fragilisation [1-7].

L'essai biaxial de disques sous pression peut être utilisé pour évaluer aisément les propriétés mécaniques en traction des matériaux en feuilles.

Cette technique expérimentale est de même nature que les essais de gonflement hydraulique comme l'essai Jovignot [14]; toutefois, la pressurisation par un gaz permet des caractérisations de matériaux à grande vitesse et/ou à haute température, ce qui est difficile à réaliser avec un système hydraulique de mise en charge.

Bien que les résultats des essais de disques soient significatifs et intéressants [8-10], ils ne sont restés que qualitatifs ou comparatifs jusqu'à maintenant; les résultats actuels sont suffisants pour révéler différents phénomènes ou mécanismes ou pour sélectionner des matériaux en vue d'une utilisation déterminée; en revanche, des résultats quantitatifs manquent pour déterminer les lois de comportement ou d'endommagement qui sont nécessaires aux calculs des structures mécaniques. Bien sûr, les essais de traction donnent les propriétés mécaniques pour dimensionner les structures mais leur sensibilité est plus faible que celle des essais de disques notamment dans le cas de matériaux en feuilles et les essais de traction uniaxiale ne représentent pas le comportement d'une paroi mince soumise à une pression de gaz.

Pour ces raisons, nous avons développé une méthode analytique pour déterminer les propriétés mécaniques intrinsèques du disque ; cette méthode complète et améliore les nombreuses approches théoriques réalisées depuis plus de 40 ans pour déterminer les contraintes et les déformations dans une membrane gonflée par un fluide sous pression [11-14].

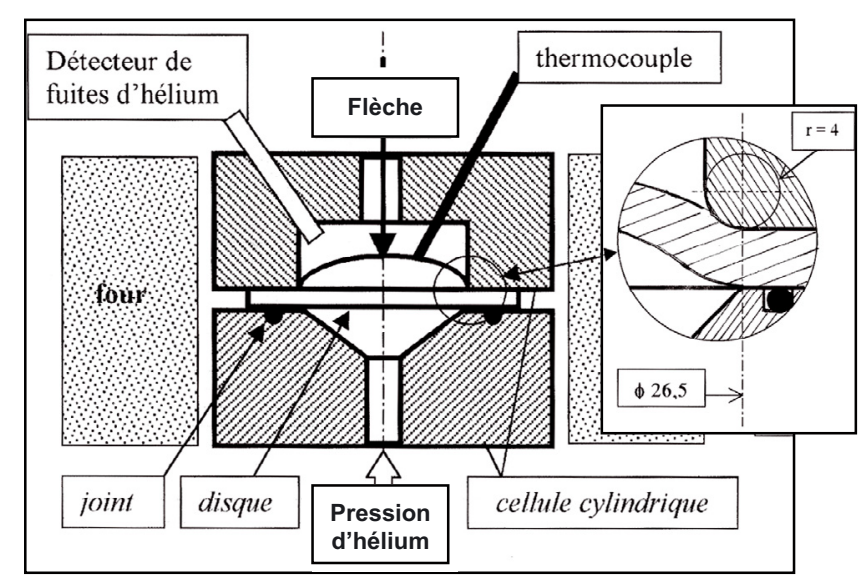

Pressurisation de $10^{-2}$ à $2 \times 10^{4} \mathrm{MPa} \cdot \mathrm{min}^{-1}-$ Température de 20 à $900{ }^{\circ} \mathrm{C}$

Fuite détectable au travers du disque : $1 \mathrm{~N} . \mathrm{cm}^{3} \cdot \mathrm{an}^{-1}$

Le disque est gonflé par une pression d'hélium. Pendant l'essai sont enregistrés la pression $P$, la flèche $W$ au pôle, la température, le débit d'hélium au travers du disque.

Fig. 1. Cellule d'essai biaxial de disques.

\section{Technique expérimentale des essais de traction biaxiale}

\subsection{Rupture de disques}

Avant essai, l'échantillon le plus courant est un disque plat de diamètre $58 \mathrm{~mm}$ et d'épaisseur $0,75 \mathrm{~mm}$. Le disque est encastré à partir d'un diamètre de $26,50 \mathrm{~mm}$ entre les deux flasques cylindriques en acier de la cellule d'essai (Fig. 1) ; ce disque est déformé par une pression continûment croissante de gaz neutre jusqu'à sa rupture ou sa fissuration finale.

La cellule est normalement étanchée par un joint torique en élastomère; un joint torique métallique est utilisé lorsque la température dépasse $150{ }^{\circ} \mathrm{C}$ ou lorsque des conditions de pureté du gaz l'exigent.

De l'hélium est stocké dans une bouteille dont la pression peut atteindre $150 \mathrm{MPa}$. La pressurisation du disque est réalisée à vitesse constante par l'intermédiaire d'actionneurs pneumatiques contrôlés par un microordinateur [15]; les équipements automatisés permettent de réaliser des essais mécaniques à vitesse de déformation comprise entre $10^{-6}$ et $10^{0} \mathrm{~s}^{-1}$ (vitesse de pressurisation de $10^{-2}$ à $2 \times 10^{4} \mathrm{MPa} \cdot \mathrm{min}^{-1}$ ) ; la sollicitation mécanique imposée par le gaz présente donc une gamme étendue de vitesses (6 décades) classiquement inaccessibles avec un seul équipement.

La pression $P$ de l'hélium définit le chargement mécanique et la flèche $W$ au pôle du disque traduit la déformation macroscopique; ces deux paramètres sont mesurés en continu jusqu'à la rupture de l'éprouvette [16].

La cellule et le disque peuvent être chauffés jusqu'à $900{ }^{\circ} \mathrm{C}$ grâce à un four annulaire; la température de l'éprouvette est contrôlée par un thermocouple en contact avec le disque. 
De très faibles fuites d'hélium au travers de l'échantillon peuvent être détectées grâce à un spectromètre de masses analysant l'atmosphère au-dessus du disque.

\subsection{Fluage de disques}

La cellule d'essai et le disque sont chauffés jusqu'à la température d'essai ; puis la cellule est pressurisée rapidement à la pression nominale de l'essai; cette pression est ensuite maintenue constante jusqu'à la rupture du disque. La pression $P$ et la flèche $W$ sont enregistrées en continu.

\subsection{Fatigue de disques}

Des disques identiques et des cellules légèrement modifiées sont utilisés pour réaliser des essais de fatigue; des pressions cycliques $P_{0}$ et $P_{1}$ sont appliquées sur les faces inférieure et supérieure du disque; le chargement mécanique est donné par la différence $\left|P_{0}-P_{1}\right|$ qui crée une déformée de flèche $W$ alternativement vers le haut et vers le bas. Le cycle dure environ $30 \mathrm{~s}$.

\section{Calcul des propriétés mécaniques d'un disque sous pression}

\subsection{Méthode de calcul}

\subsection{1 État de contrainte et de déformation au pôle du disque}

Près de son pôle, le disque déformé en coupole est soumis aux contraintes $\sigma_{\varphi}$ et $\sigma_{\theta}$ suivant un méridien et une circonférence et à la contrainte radiale $\sigma_{z}$ dans le sens de son épaisseur.

Seule la sollicitation du disque en traction sera déterminée car la flexion peut être négligée pour une membrane peu épaisse. La contrainte $\sigma_{z}$ est égale à la pression $P$ du gaz sur la face sollicitée; cette contrainte est négligeable car elle ne vaut que quelques pourcents des contraintes $\sigma_{\varphi}$ et $\sigma_{\theta}$ (sollicitation en membrane).

Près de son pôle, le disque est donc soumis à un état de contraintes planes et son épaisseur diminue de $e_{0}$ à $e$; la déformation $\varepsilon_{z}$ suivant l'épaisseur du disque est donc égale à $\operatorname{Ln}\left(e / e_{0}\right)$; cette déformation est négative.

\subsubsection{Hypothèses de calcul}

- Le matériau est homogène et isotrope.

- Dans un premier temps, la variation du rayon de courbure dans l'épaisseur du disque est négligée.

- Le disque se déforme en membrane.

- Déformations au pôle du disque dans son plan :

$$
\varepsilon_{\varphi}=\varepsilon_{\theta}
$$

- Rayons de courbure au pôle du disque déformé en coupole :

$$
\rho_{\varphi}=\rho_{\theta}=\rho
$$

- Contraintes au pôle du disque dans son plan :

$$
\sigma_{\varphi}=\sigma_{\theta}=(P \rho) /(2 e)
$$

$\rho$ rayon au pôle du disque déformé, $e$ épaisseur au pôle du disque déformé, $P$ pression du gaz.

- Le volume d'un matériau métallique est constant au cours d'une déformation plastique :

$$
\varepsilon_{\varphi}+\varepsilon_{\theta}+\varepsilon_{z}=0
$$

\subsubsection{Contrainte et déformation équivalentes}

La contrainte $\bar{\sigma}$ et la déformation $\bar{\varepsilon}$ équivalentes de von Mises sont déterminées dans le but de comparer les propriétés mécaniques obtenues avec des disques et d'autres éprouvettes.

$$
\begin{aligned}
& \bar{\sigma}=\frac{1}{\sqrt{2}}\left[\left(\sigma_{\phi}-\sigma_{\theta}\right)^{2}+\left(\sigma_{\theta}-\sigma_{z}\right)^{2}+\left(\sigma_{z}-\sigma_{\phi}\right)^{2}\right]^{1 / 2} \\
& \bar{\varepsilon}=\frac{\sqrt{2}}{3}\left[\left(\varepsilon_{\phi}-\varepsilon_{\theta}\right)^{2}+\left(\varepsilon_{\theta}-\varepsilon_{z}\right)^{2}+\left(\varepsilon_{z}-\varepsilon_{\phi}\right)^{2}\right]^{1 / 2} \\
&(3)+\sigma_{z} \approx 0 \Rightarrow \bar{\sigma}=\sigma_{\varphi}=\sigma_{\theta} \\
& \Rightarrow \bar{\sigma}=\frac{P \rho}{2 e} \\
&(1)+(4)+\varepsilon_{z}=\operatorname{Ln}\left(e / e_{0}\right) \\
& \Rightarrow \bar{\varepsilon}=2 \varepsilon_{\varphi}=2 \varepsilon_{\theta} \\
& \Rightarrow \bar{\varepsilon}=\operatorname{Ln}\left(e_{0} / e\right)
\end{aligned}
$$

$P$ pression du gaz, $\rho$ rayon de courbure au pôle du disque déformé, e épaisseur au pôle du disque déformé, $e_{0}$ épaisseur du disque avant essai.

\subsubsection{Charge unitaire et allongement}

Souvent, les propriétés mécaniques en traction ne sont pas données sous forme rationnelle, contrainte $\sigma$ et déformation $\varepsilon$, mais sous forme conventionnelle, charge unitaire et allongement.

\section{- Charge unitaire équivalente $R$}

En traction uniaxiale, la charge unitaire est égale à la force $F$ de traction divisée par la section initiale $S_{0}$ de l'éprouvette; la charge unitaire $F / S_{0}$ passe par un maximum au moment où apparaît la striction.

Pour l'essai de traction biaxiale de disques, la contrainte équivalente $\bar{\sigma}$ est égale à une force linéique $F_{1}$ $\left(F_{1}=P \rho / 2\right)$ divisée par l'épaisseur $e$ du disque déformé; par analogie avec l'essai de traction, la charge unitaire $R$ équivalente est égale à la même force linéique $F_{1}$ divisée par l'épaisseur $e_{0}$ initiale du disque.

$$
\Rightarrow R=\frac{P \rho}{2 e_{0}}
$$




\section{- Allongement relatif équivalent $A$}

En traction uniaxiale, la déformation $\varepsilon$ est calculée à partir de l'allongement relatif $A=\Delta L / L_{0}$ de l'éprouvette, $\Delta L$ étant son allongement absolu et $L_{0}$ sa longueur initiale :

$$
\varepsilon=\operatorname{Ln}(1+A) \Rightarrow A=\exp (\varepsilon)-1
$$

Pour l'essai biaxial de disques, l'allongement est calculé à partir de la déformation par la même relation qu'en traction; comme la déformation dépend des épaisseurs du disque avant et pendant l'essai par la relation (6), nous pouvons en déduire la valeur de l'allongement du matériau :

$$
A=\frac{e_{0}}{e}-1
$$

\subsection{Détermination des rayons et des épaisseurs}

Pour déterminer les propriétés mécaniques du disque, il est nécessaire de connaître le rayon $\rho$ de courbure et l'épaisseur $e$ au pôle au cours de la déformation.

\subsubsection{Déformée du disque}

Si la déformée du disque est une coupole sphérique, le rayon $\rho$ de courbure au pôle est égal au rayon de la sphère; ce rayon peut être déterminé en fonction de la flèche $W$ par la relation géométrique (9) obtenue pour la fibre extérieure.

$$
\rho=\left(W^{2}+A^{2}\right) /(2 W)-r
$$

$W$ flèche au pôle du disque, $A$ rayon intérieur de la zone encastrée du disque (Fig. 2), $r$ rayon de l'enclume à l'encastrement aval (Fig. 2).

Des mesures dimensionnelles ont permis de vérifier l'hypothèse de la déformée sphérique.

\subsubsection{Déformation et épaisseur du disque}

Des calculs analytiques de plasticité permettent d'obtenir les déformations en fonction de la flèche $W$ mesurée.

$\mathrm{Au}$ pôle du disque déformé en coupole et au niveau de sa fibre moyenne, un incrément de déformation $\mathrm{d} \varepsilon_{\phi}$ (ou $\mathrm{d} \varepsilon_{\theta}$ ) suivant un méridien (ou une circonférence) est égal à la variation relative $\mathrm{d} \rho / \rho$ du rayon de la coupole; en considérant que le disque ne se déforme qu'à partir de son point de tangence $D$ avec l'outillage (Fig. 2), la variation $\mathrm{d} \rho \mathrm{du}$ rayon est égale à la variation $\mathrm{d} h$ de la hauteur de la coupole déformable.

La déformation $\varepsilon_{\phi i}$ suivant un méridien est obtenue en intégrant les incréments $\mathrm{d} \varepsilon_{\phi}$ de déformation correspondant à des incréments $\mathrm{d} W$ de la flèche au pôle depuis l'état non déformé $(W=0)$ jusqu'à une valeur déterminée $W_{i}$ de la flèche.

$$
\varepsilon_{\varphi i}=\int_{0}^{W i} \frac{\mathrm{d} h}{\rho} \text { et } \bar{\varepsilon}_{i}=2 \varepsilon_{\varphi i}
$$

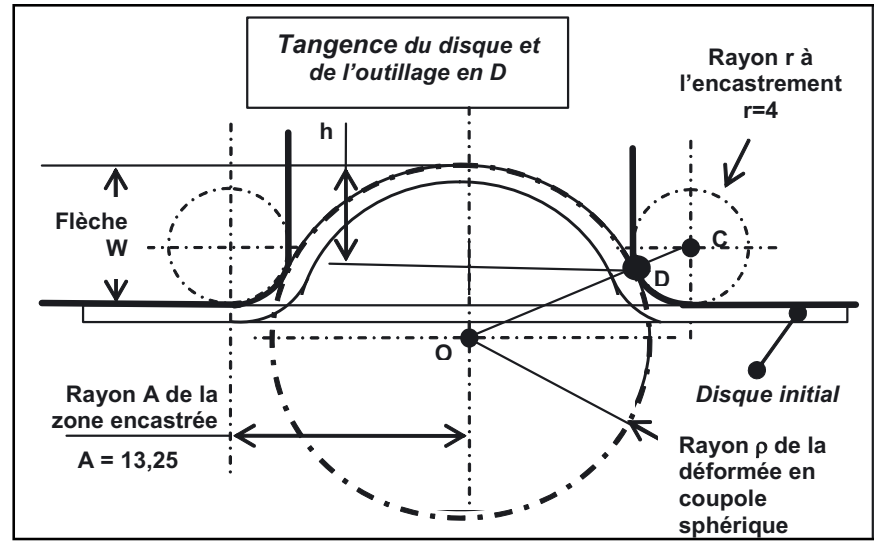

Par hypothèse, la déformée du disque est une coupole sphérique tangente à l'outillage au point $D$.

Fig. 2. Déformée du disque - paramètres géométriques.

En utilisant les relations géométriques entre les différents paramètres montrés par la figure 2, la déformation équivalente est calculée pour différentes valeurs $W_{i}$ de la flèche au pôle grâce à la formule (10) :

$$
\bar{\varepsilon}_{i}=2 \int_{0}^{W_{i}\left[1-\frac{2 r}{A}\left(\frac{1}{1+\frac{W^{2}}{A^{2}}}\right) \sin \left(2 \operatorname{Arctg}\left(\frac{W}{A}\right)\right)\right]} \mathrm{d} \frac{}{\rho} W
$$

$W$ flèche au pôle du disque, $A$ rayon intérieur de la zone encastrée du disque, $r$ rayon de l'enclume à l'encastrement aval, $\rho$ rayon de la déformée en coupole sphérique - $\rho$ est calculé en fonction de $W$ par la relation (9).

Les déformations calculées pour différentes flèches $W_{i}$ à partir de la formule (10) permettent de déterminer l'épaisseur au pôle $e_{i}$ des disques déformés grâce à la relation (6) ; le calcul des déformations et des épaisseurs a été validé par la mesure des épaisseurs au pôle sur des disques de plusieurs matériaux déformés en coupole de différentes hauteurs $W_{i}$.

\subsection{Fatigue de disques}

Pendant les essais de fatigue, les flèches $W$ sont faibles si bien que les déformations sont maximales au niveau de l'encastrement; ainsi, en fatigue oligocyclique, le matériau est plastifié à l'encastrement du disque alors qu'il n'est sollicité que dans le domaine d'élasticité près du pôle. Les calculs précédents (Sects. 3.1 et 3.2) ne sont plus valables pour la fatigue car ils déterminent les propriétés au pôle du disque.

En fatigue oligocyclique, le nombre $N$ de cycles à rupture dépend de la déformation plastique $\Delta \varepsilon_{\mathrm{p}}$ par une loi de Manson-Coffin [19] :

$$
\Delta \varepsilon_{\mathrm{p}}=A N^{-a}
$$


Si un essai de rupture monotone (déformation $\varepsilon_{R}$ à la rupture) est considéré comme un essai de fatigue en 0,5 cycle, la loi de Manson-Coffin devient :

$$
\Delta \varepsilon_{\mathrm{p}}=\varepsilon_{R}(N / 0,5)^{-a}
$$

$\mathrm{Au}$ début de la pressurisation, la déformation et la contrainte à l'encastrement sont calculées à partir de la flèche $W$ grâce aux formules analytiques pour des membranes sous pression dans le domaine d'élasticité [20]; ainsi la flèche $W_{\mathrm{p}}$ est déterminée lorsque la limite d'élasticité $R_{\mathrm{p}}$ est atteinte.

La déformation plastique $\Delta \varepsilon_{\mathrm{p}}$ dépend de la différence de flèche $\left(W-W_{\mathrm{p}}\right)$ pour laquelle $W$ est la flèche mesurée au cours de l'essai; pour les faibles flèches, la relation entre la déformation et la flèche est donnée par une approximation linéaire :

$$
\Delta \varepsilon_{\mathrm{p}}=K\left(W-W_{\mathrm{p}}\right)
$$

Pour la fatigue de disques, la flèche $W$ et le nombre $N$ de cycles à rupture sont reliés par la loi empirique (14) :

$$
\left(W-W_{\mathrm{p}}\right)=C N^{-c}
$$

Le coefficient $K$ peut être calculé à partir des formules (11) à (14).

\section{Résultats expérimentaux}

Des résultats expérimentaux sont présentés pour montrer le champ d'application et l'intérêt de l'essai de disques associé au calcul des propriétés mécaniques; pour ces raisons, les propriétés du matériau et les phénomènes métallurgiques ne sont décrits ou analysés que succinctement.

\section{Matériau étudié}

Le matériau étudié est un cuivre faiblement allié avec notamment du chrome. Ce matériau a subi un traitement préalable de durcissement structural; les précipités sont d'abord mis en solution à haute température puis une hypertrempe permet de conserver une solution solide sursaturée à température ambiante; un revenu du matériau hypertrempé amène un durcissement important grâce à une précipitation fine et cohérente à partir de la solution solide sursaturée.

\subsection{Rupture monotone de disques}

Pour un matériau métallique, la rationalisation d'un essai mécanique ne peut être réalisée facilement que si la déformation est uniforme dans le volume de matériau considéré; ainsi en traction, la rationalisation est difficile à obtenir lorsque la striction est apparue.

En traction, le début de la striction est facilement repéré car il correspond au maximum de la force (ou de la charge unitaire) appliquée à l'éprouvette; ce maximum est atteint pour l'allongement uniforme Au qui peut être largement inférieur à la valeur $A_{\text {r }}$ obtenue à la rupture.

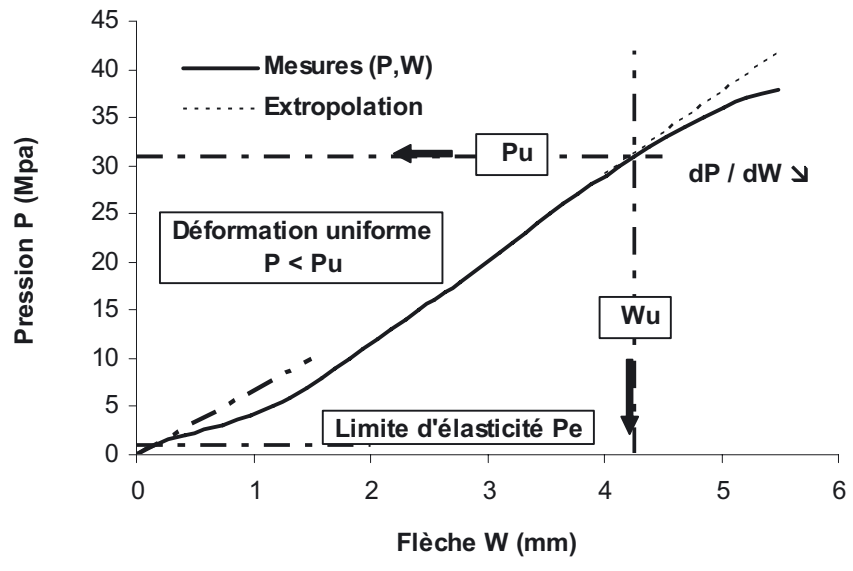

Cuivre peu allié à $20{ }^{\circ} \mathrm{C}$

La courbe s'infléchit à partir de la flèche $W_{\mathrm{u}}$ lorsque la déformation du matériau n'est plus homogène.

Fig. 3. Courbe pression-flèche.

\subsubsection{Courbe expérimentale pression-flèche de l'essai de disques}

Pour l'essai de disques, les courbes expérimentales ne présentent pas de maximum de la pression avant la rupture finale (Fig. 3) mais seulement une diminution de la pente; cet infléchissement à partir de la pression $P_{\mathrm{u}}$ et de la flèche $W_{\mathrm{u}}$ correspond à la fin de la déformation uniforme du matériau; une déformation localisée apparaît et la déformée du disque n'est plus la coupole sphérique idéale.

\subsubsection{Domaine de validité des calculs des propriétés mécaniques}

À partir de la courbe expérimentale (Fig. 3), la charge unitaire équivalente $R$ est calculée grâce aux formules (7) et $(9)$.

La charge unitaire $R$ a été représentée en fonction de la flèche $W$ (Fig. 4) ; les résultats montrent plusieurs domaines définissant la validité du calcul des propriétés mécaniques.

\section{- Début de la déformation plastique à la flèche $W_{p}$}

Pour les flèches inférieures à $1 \mathrm{~mm}$, les résultats sont incohérents car la charge diminue alors que le disque est de plus en plus déformé : les hypothèses du calcul ne sont pas valides dans ce cas.

Des observations de disques montrent que, pour les faibles flèches, le matériau est plastifié à l'encastrement du disque et que la déformation au pôle est restée élastique; comme la déformation est très hétérogène, le rayon au pôle du disque est différent de celui calculé pour une sphère.

La relation (10) permet de calculer la flèche $W_{\mathrm{p}}$ nécessaire pour obtenir une déformation plastique égale à $0,2 \%$. 


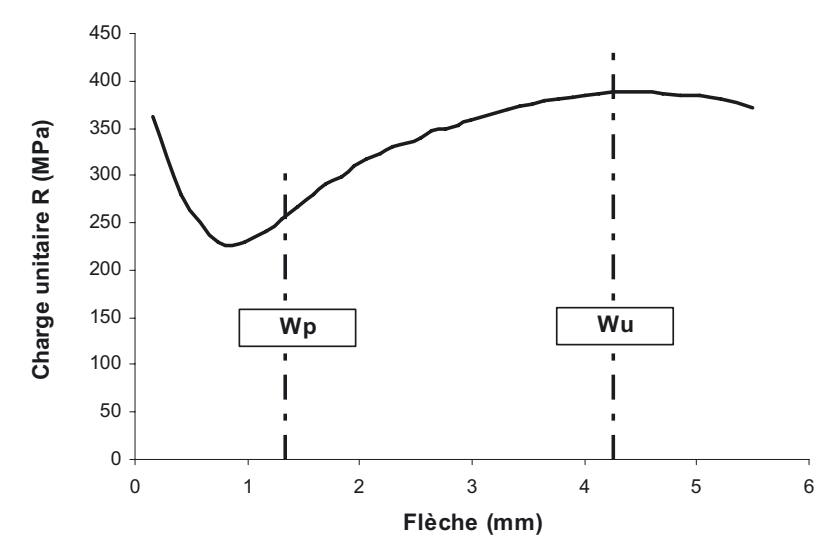

Cuivre peu allié à $20^{\circ} \mathrm{C}$

Pour des flèches inférieures à $1 \mathrm{~mm}$, la charge calculée n'est pas correcte car les hypothèses du calcul ne sont pas valides. La charge atteint un maximum à la flèche $W_{\mathrm{u}}$ lorsque la déformation n'est plus homogène.

Fig. 4. Charge unitaire en fonction de la flèche.

\section{- Fin de la déformation plastique uniforme à la flèche $W_{u}$}

La charge unitaire $R$ croît pour atteindre un maximum lorsque la flèche est égale à la valeur $W_{\mathrm{u}}$. Pour les plus grandes flèches, la charge diminue lorsqu'apparaissent des zones de striction

NB. La flèche $W_{u}$ de déformation uniforme calculée pour le maximum de la charge unitaire est identique à celle trouvée pour l'infléchissement de la courbe expérimentale (Fig. 3); mais la flèche $W_{u}$ est plus facile à déterminer pour le maximum de la charge que pour l'infléchissement de la courbe pression-flèche.

\subsubsection{Propriétés mécaniques conventionnelles}

Quand les hypothèses de calcul sont valides (flèches de $W_{\mathrm{p}}$ à $W_{\mathrm{u}}$ ), la charge unitaire $R$ et l'allongement $A \mathrm{du}$ disque sont déterminés à partir des formules (7)-(10).

Le calcul analytique des propriétés mécaniques du disque donne des résultats cohérents puisque nous retrouvons les caractéristiques de traction (Fig. 5) :

- la première valeur de la charge unitaire calculée pour la flèche $W_{\mathrm{p}}$ correspondant à une déformation plastique de $0,2 \%$ est identique à la limite conventionnelle d'élasticité $R_{\mathrm{p}}$ obtenue en traction uniaxiale pour la même déformation de $0,2 \%$,

- pour la flèche $W_{\mathrm{u}}$, la charge et l'allongement du matériau atteignent des maxima qui sont identiques aux valeurs de la résistance $R_{\mathrm{m}}$ et de l'allongement uniforme $A_{\mathrm{u}}$ obtenus en traction uniaxiale.

\subsubsection{Propriétés mécaniques rationnelles et loi d'écrouissage}

Dans le domaine de validité (flèches de $W_{\mathrm{p}}$ à $W_{\mathrm{u}}$ ), la contrainte $\bar{\sigma}$ et la déformation $\bar{\varepsilon}$ du disque sont calculées

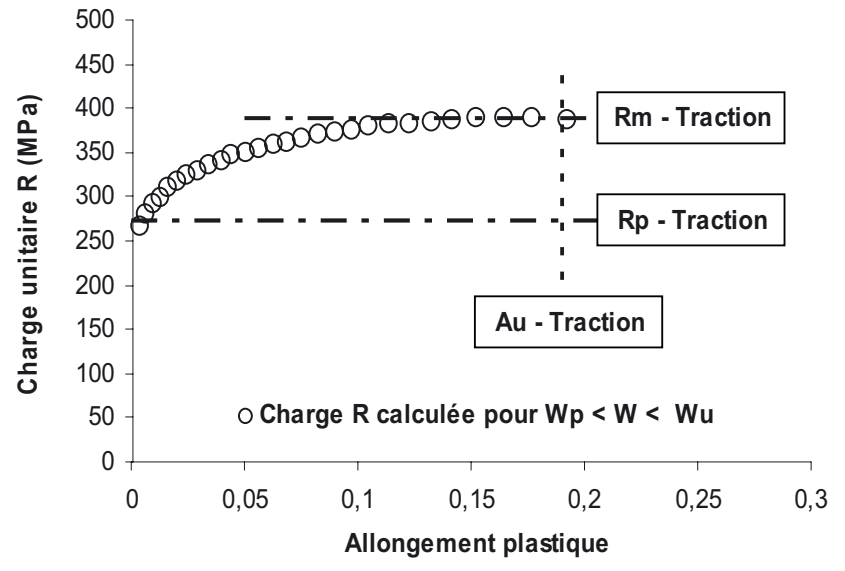

Cuivre peu allié à $20^{\circ} \mathrm{C}$

Les propriétés mécaniques conventionnelles sont identiques pour la traction et pour le disque.

Fig. 5. Propriétés mécaniques conventionnelles.

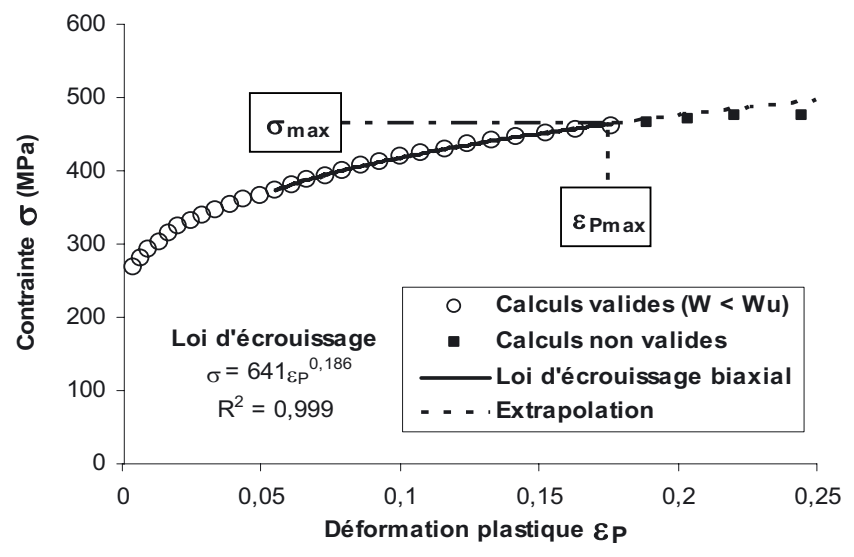

Cuivre peu allié à $20^{\circ} \mathrm{C}$

Comme en traction, l'indice d'écrouissage (exposant de la loi d'écrouissage) est égal à la déformation maximale.

Fig. 6. Propriétés mécaniques rationnelles.

à partir des formules (5), (6), (9) et (10). Les résultats (Fig. 6) permettent de vérifier que l'écrouissage suit la loi puissance proposée par Hollomon [17].

L'exposant $n$ de la loi puissance est défini comme l'indice d'écrouissage; en traction, on démontre que l'indice $n$ d'écrouissage est égal à la déformation uniforme maximale $\varepsilon_{\max }$. Pour l'essai de disques, le même type de résultat est trouvé puisque l'indice d'écrouissage est égal à la déformation maximale (Fig. $6: n=0,186$ \# $\left.\varepsilon_{\max }=0,175\right)$ mais avec une précision moins bonne qu'en traction.

La méthode de calcul des propriétés du disque et le domaine de validité des calculs sont à nouveau validés.

NB. Si la fin de la déformation homogène n'était pas déterminée, les propriétés mécaniques seraient calculées jusqu'à la rupture. Comme, aux plus grandes flèches, les résultats ne sont pas valides, ils n'appartiennent 


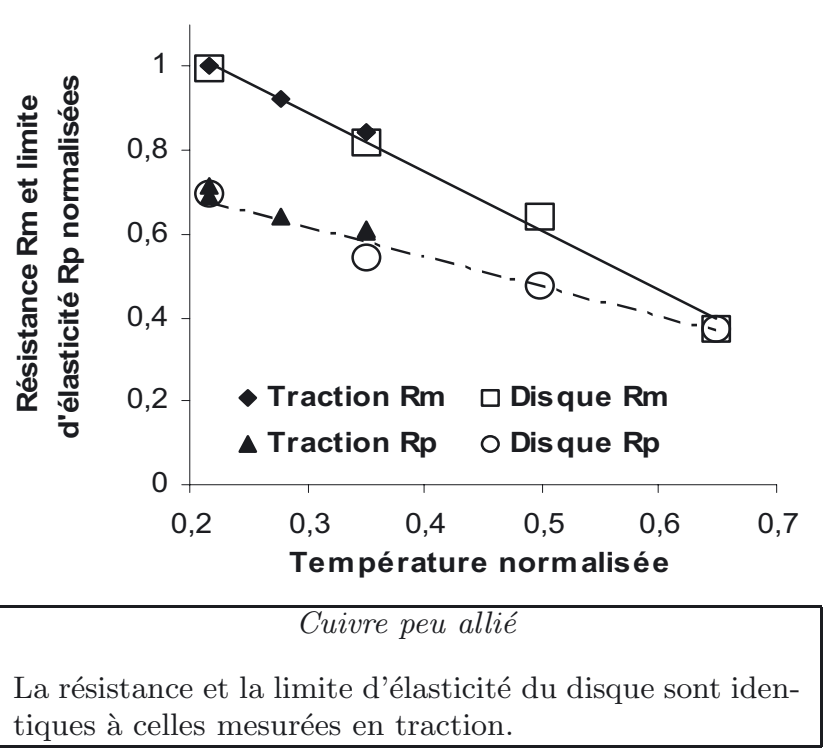

Fig. 7. Résistance mécanique en fonction de la température.

pas à la loi d'écrouissage trouvée pour le matériau homogène (Fig. 6). De plus, si les résultats obtenus aux grandes flèches étaient utilisées pour déterminer la loi d'écrouissage, le coefficient $n$ d'écrouissage serait différent de la déformation maximale.

\subsubsection{Influence de la température}

À faible vitesse de déformation, la résistance $R_{\mathrm{m}}$, la limite d'élasticité $R_{\mathrm{p}}$ et l'allongement uniforme $A_{\mathrm{u}}$ sont identiques pour un disque ou une éprouvette de traction cylindrique issus de la même barre (Figs. 7 et 8). Les faibles dispersions sont plus dues aux hétérogénéités du matériau qu'aux méthodes d'essais puisque, par exemple, l'allongement uniforme du disque appartient à l'intervalle de dispersion obtenu en traction.

Les essais de disques ont été réalisés jusqu'à la température devant être atteinte en service. Les essais de traction ont été réalisés à température beaucoup plus faible car un extensomètre mécanique était placé dans l'enceinte chauffée.

La résistance $R_{\mathrm{m}}$ baisse lorsque la température augmente ; cette baisse peut être due à un adoucissement du matériau par recristallisation ou à un phénomène de survieillissement.

Lorsque la température augmente, l'allongement $\mathrm{Au}$ baisse également; des déformations localisées s'amorcent sur des hétérogénéités du matériau; ces hétérogénéités apparaissent d'autant plus facilement que la température est élevée.

\subsubsection{Influence de la vitesse de sollicitation}

Des essais de traction ont été conduits sur des éprouvettes cylindriques avec des vitesses de déplacement de la traverse dans un rapport de 1 à 100 ; les disques ont

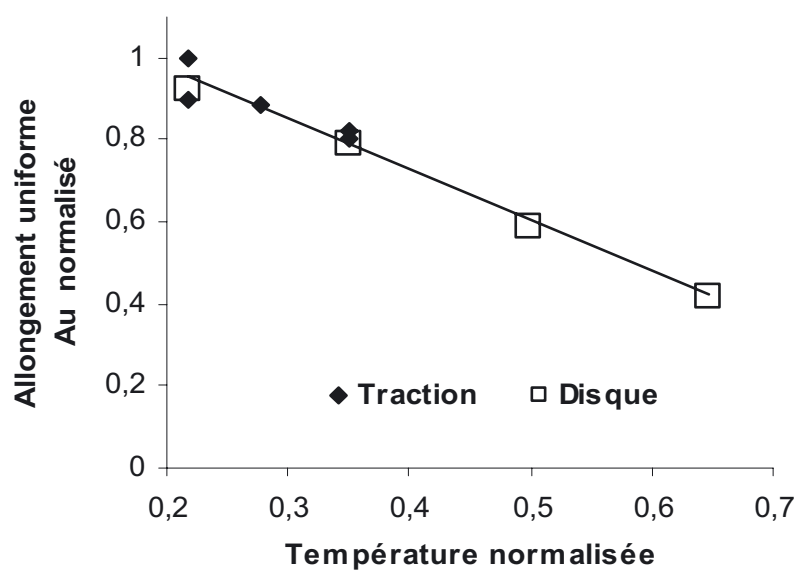

Cuivre peu allié

L'allongement du disque est identique à celui mesuré en traction.

Fig. 8. Allongement en fonction de la température.

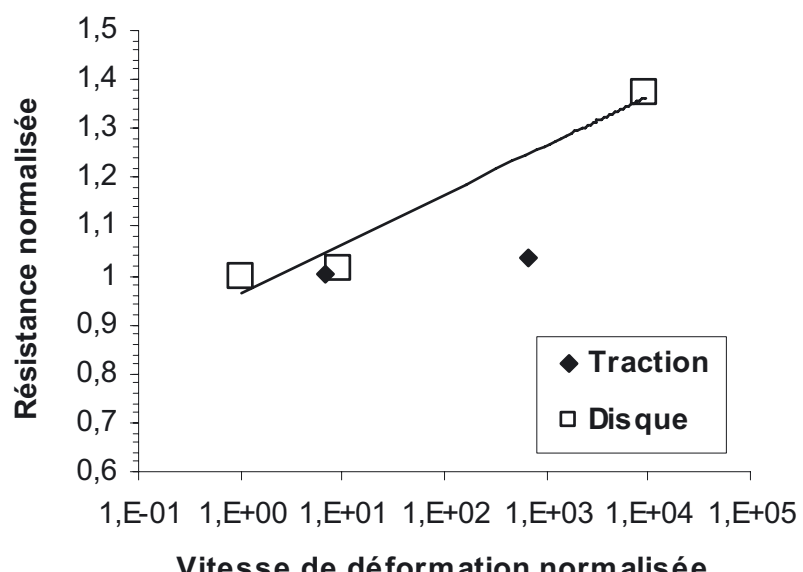

Cuivre peu allié à $20^{\circ} \mathrm{C}$

Le matériau a un comportement viscoplastique car sa résistance augmente comme la vitesse de sollicitation.

Fig. 9. Résistance mécanique à température ambiante.

été pressurisés avec des vitesses dans un rapport de 1 à 10000 .

Malgré le faible nombre de résultats à $20{ }^{\circ} \mathrm{C}$, nous retrouvons le comportement habituel des matériaux viscoplastiques dont la résistance diminue lorsque la vitesse de déformation augmente (Fig. 9).

Pour les essais les plus lents, la résistance mécanique est identique qu'elle soit mesurée avec l'essai de disque ou de traction.

Pour les essais aux plus grandes vitesses, la résistance de l'éprouvette de traction est inférieure à celle du disque. Cette différence peut s'expliquer par des effets de bords : en traction, les bords de l'éprouvette sont sous contrainte et un défaut de surface (seconde phase, défaut d'usinage... ) concentre la contrainte et amorce la striction à charge relativement faible. 


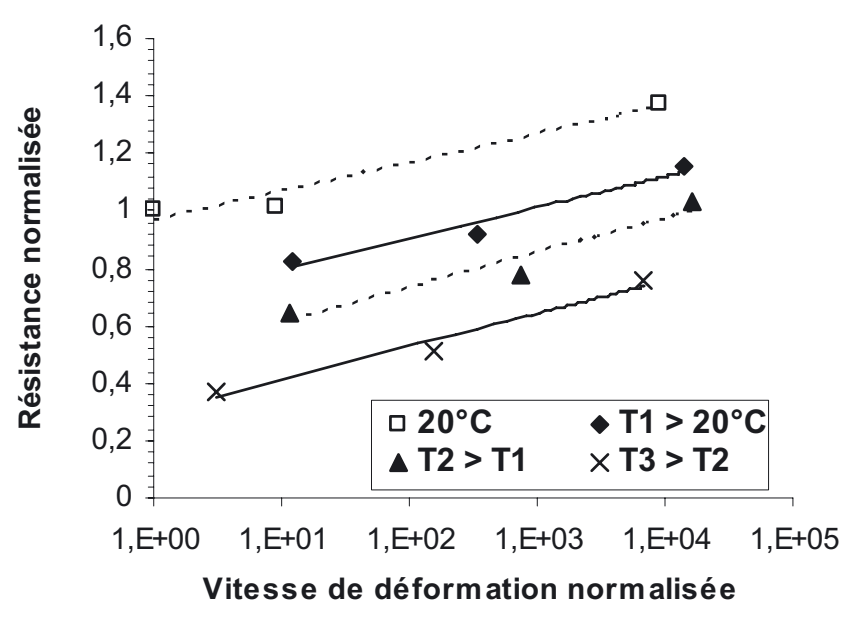

Cuivre peu allié à $20^{\circ} \mathrm{C}$

Les essais de disques peuvent être réalisés dans des gammes très larges de température et de vitesse.

Fig. 10. Résistance mécanique à différentes vitesses et températures.

À grande vitesse, le défaut de surface agit comme l'entaille d'une éprouvette de résilience.

Comme le disque est encastré, ses bords et leurs défauts sont en dehors de la zone déformée si bien qu'ils ne sont pas sollicités.

L'absence d'effet de bords permet d'utiliser les essais de disques à des températures et des vitesses élevées (Fig. 10) pour lesquelles les résultats de traction sont souvent trop dispersés pour être exploitables.

\subsection{Fluage de disques}

\subsubsection{Chargement mécanique du disque}

Le fluage de disques est réalisé en maintenant une pression constante; à suffisamment haute température, la flèche $W$ augmente si bien que le rayon $\rho$ de la coupole diminue ; la charge unitaire $R$ et la contrainte $\bar{\sigma}$ peuvent être calculées à chaque instant avec les formules (5), (7), (9) et (10); la contrainte $\bar{\sigma}$ est à peu près constante pendant tout l'essai (Fig. 11).

\subsubsection{Fluage d'un acier austénitique}

Les déformations sont calculées à partir des formules (9) et (10); l'évolution de la déformation (Fig. 12) montre les trois phases habituelles du fluage restauration [18] :

- le fluage primaire pour lequel la vitesse de déformation $\dot{\varepsilon}$ diminue;

- le fluage secondaire pour lequel la déformation se poursuit à vitesse stabilisée $\dot{\varepsilon}_{\mathrm{II}}$;

la vitesse de fluage secondaire est calculée par une régression linéaire des points expérimentaux;

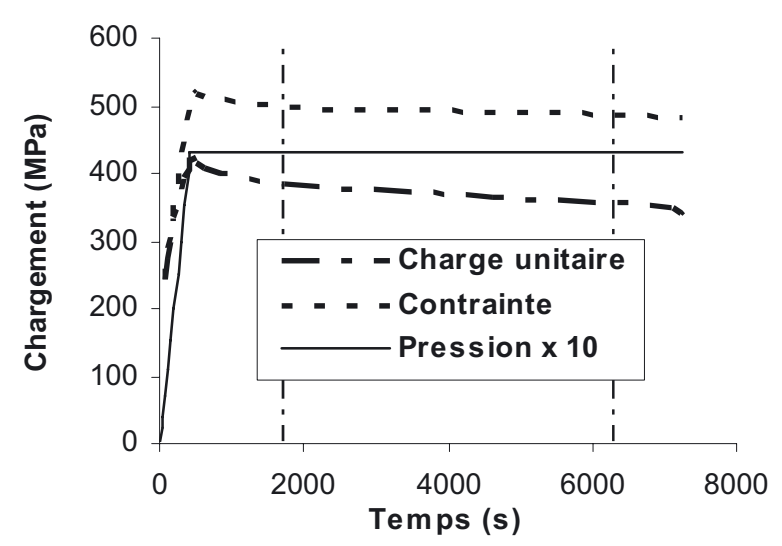

Pendant un essai de fluage de disque, la contrainte appliquée au matériau est quasi constante.

Fig. 11. Chargement d'un disque en essai de fluage.

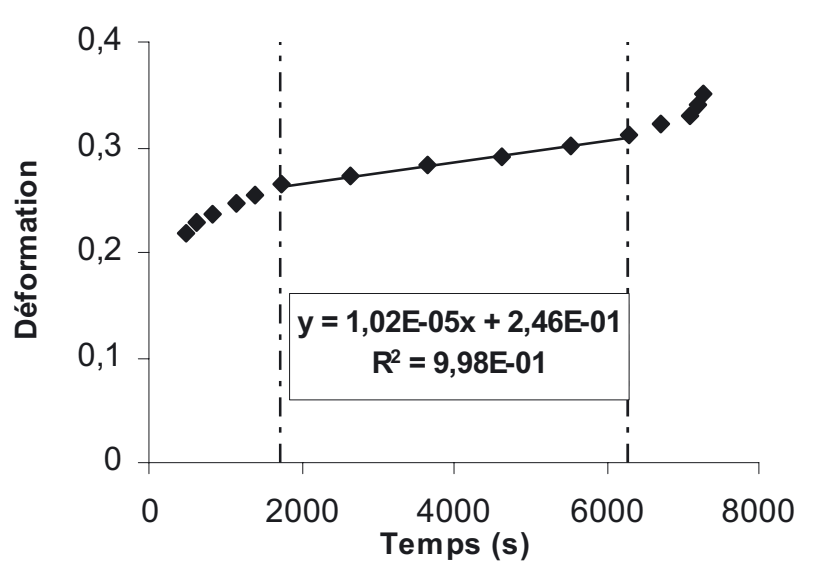

Acier austénitique

Pour un acier austénitique, on observe les 3 phases habituelles du fluage avec notamment le fluage secondaire à vitesse constante.

Fig. 12. Fluage de disques.

- le fluage tertiaire pour lequel la vitesse de déformation augmente jusqu'à la rupture finale de l'éprouvette.

\subsubsection{Fluage du cuivre peu allié}

Les courbes de fluage (Fig. 13) des disques en cuivre peu allié montrent que tous les essais se sont terminés avant d'atteindre le fluage tertiaire; en effet, une fissuration traversante se crée pendant le fluage secondaire; cette fissuration crée des fuites si bien que le disque ne peut plus être maintenu sous pression constante. Les fissures sont détectées alors que leur longueur est faible; cet endommagement ne serait révélé que dans un stade ultérieur par le fluage d'éprouvettes plus massives. 

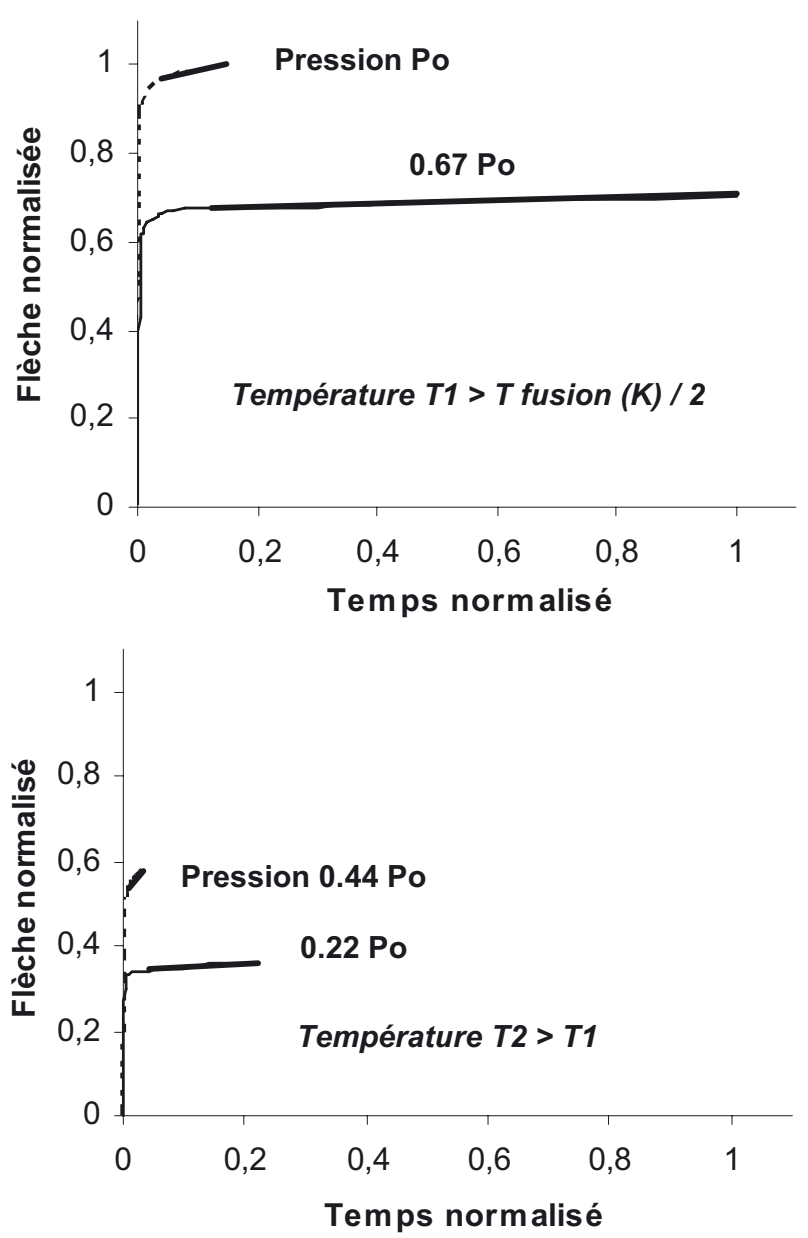

Cuivre peu allié

Tous les essais de fluage de disques en cuivre se sont arrêtés pendant la $2^{\mathrm{e}}$ phase lorsque sont apparues des fissures traversantes rendant impossible le maintien sous pression.

Fig. 13. Fluage de disques.

\subsubsection{Observations micrographiques du cuivre peu allié}

Des micrographies mettent en évidence des fissures intergranulaires dans les disques en cuivre peu allié déformés à haute température (Fig. 14b) ; dans les zones non déformées des disques chauffés, les joints de grains sont devenus relativement épais (Fig. 14a).

\subsection{Fatigue de disques}

Plusieurs types d'éprouvettes en cuivre peu allié ont été sollicités en fatigue :

- des disques (épaisseur égale à $0,75 \mathrm{~mm}$ ) sont pressurisés alternativement et symétriquement sur chacune de leurs faces,

- des éprouvettes cylindriques (diamètre égal à $8 \mathrm{~mm}$ ) sont soumises à des allongements cycliques symétriques en traction-compression,

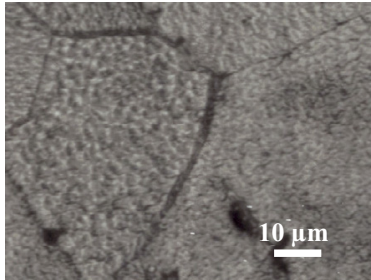

A - Zone non déformée

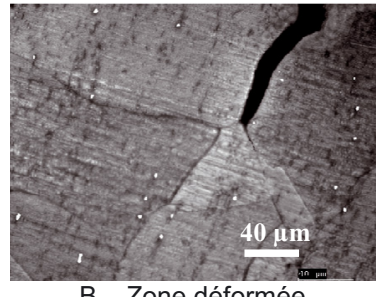

B - Zone déformée
Cuivre peu allié

Après chauffage, les joints de grains deviennent épais ; les fissures se créent dans ces joints épais pendant l'essai de disques.

Fig. 14. Micrographies de disques essayés en fluage à haute température.

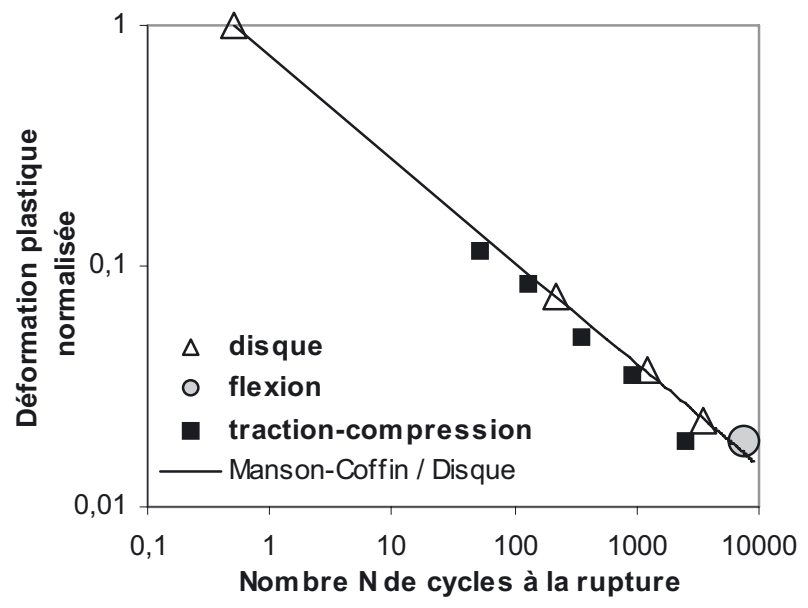

Cuivre peu allié
Les durées de vie mesurées par les 3 méthodes d'essai
sont à peu près identiques.

Fig. 15. Fatigue oligocyclique.

- des lames triangulaires (épaisseur égale à $2 \mathrm{~mm}$ ) sont déformées alternativement et symétriquement en flexion.

Les résultats de fatigue des disques sont exploités par une loi de Manson-Coffin qui interpole correctement tous les points obtenus en traction-compression et en flexion (Fig. 15) : en conséquence, le calcul des propriétés mécaniques du disque en essai de fatigue (Sect. 3.3) est validé.

Les durées de vie sont légèrement différentes avec les trois types d'essais à cause des épaisseurs des éprouvettes et de la sollicitation mécanique :

- comme le matériau n'était pas parfaitement homogène et isotrope, les éprouvettes de traction-compression flambaient dès les premiers cycles si bien que les déformations étaient localisées et mal estimées,

- la fatigue de disques crée un cycle asymétrique de déformation : de $\varepsilon_{\mathrm{t}}+\varepsilon_{\mathrm{f}}$ à $\varepsilon_{\mathrm{t}}-\varepsilon_{\mathrm{f}}$ où $\varepsilon_{\mathrm{f}}$ est la déformation de flexion et $\varepsilon_{\mathrm{t}}$ de traction; le chargement est symétrique pour les autres essais, 
- l'épaisseur de l'échantillon a une influence sur l'amorçage et la propagation des fissures :

- l'amorçage est plus facile dans le disque peu épais que dans les autres éprouvettes plus massives car la concentration de contrainte sur des défauts y est plus élevée,

- les défauts des arêtes amorcent les fissures dans les éprouvettes de flexion alors qu'ils ne sont pas sollicités dans les disques,

- la phase de propagation est plus courte dans les éprouvettes les plus fines.

NB. Les dispersions obtenues entre les différents résultats de fatigue ne sont pas supérieures à celles obtenues habituellement avec un même type d'essai de fatigue oligocyclique.

\section{Discussion}

\subsection{Bilan de l'étude thermomécanique du cuivre peu allié}

Après son vieillissement initial pendant le traitement de durcissement structural, le cuivre peu allié est dans un état métastable; lorsqu'il est maintenu en température, il peut évoluer vers un état plus stable s'il y a diffusion des atomes de solutés. Il se forme alors de nouvelles phases ou bien, la morphologie et la taille des phases préexistantes peuvent évoluer.

Les micrographies montrent cette évolution métallurgique caractérisée par des phases intergranulaires quasi continues.

Les phases intergranulaires sont responsables d'une fragilisation microscopique car elles localisent la fissuration; elles amènent également une fragilisation macroscopique puisqu'elles diminuent à la fois la résistance et l'allongement uniforme du matériau. Les fissurations intergranulaires sont également à l'origine des fuites pendant le fluage.

La formation ou l'évolution des phases métallurgiques sont reliées à un phénomène de diffusion à l'état solide. À température moyenne, la diffusion des atomes est localisée au niveau des joints de grains car ils correspondent à des zones sans cohérence et cohésion du réseau cristallin; les atomes de solutés diffusent plus facilement dans ces zones perturbées; les observations micrographiques confirment la diffusion intergranulaire. La diffusion est un phénomène thermiquement activé; cette activation thermique explique que la fissuration des disques et les fuites apparaissent d'autant plus vite que la température est élevée.

Les phénomènes de diffusion peuvent également être étudiés en traction mais les dispersions des résultats dues aux effets de bord à température élevée peuvent masquer la cohérence des résultats.

De plus, le détecteur de fuites d'hélium permet de détecter l'endommagement lorsque la longueur des fissures atteint l'épaisseur du disque; des fissures aussi courtes ne seraient pas aussi facilement détectées par des essais de traction aux résultats trop dispersés.

\subsection{Applications de l'essai biaxial de disques}

Les essais de disques peuvent être appliqués à diverses installations pour lesquelles il faut caractériser l'endommagement des matériaux soumis à des cycles de déformation de grande amplitude et/ou à grande vitesse : moufle de fours pour traitements thermiques, soufflets métalliques pour compensateur de dilatation, réacteurs pour l'industrie chimique, moteurs pour l'aéronautique ou l'aérospatiale...

Notamment, la détection très sensible de fuites au travers du disque est intéressante pour caractériser l'étanchéité des parois minces de ces équipements.

L'essai de disques représente la sollicitation des structures réelles pour lesquelles des zones amincies et entourées de parties plus massives ne sont pas sujettes aux effets de bord.

Les essais de disques peuvent être également appliqués à la caractérisation des propriétés de mise en forme des métaux en feuilles à différentes vitesses; le calcul proposé pour les contraintes et les déformations permet d'identifier de façon fiable le domaine de déformation uniforme, ce qui est nécessaire pour déterminer les limites de formage du matériau. Cette information a toujours été difficile à obtenir avec les essais de gonflement hydraulique.

\subsubsection{Gammes de température et de vitesse}

Les essais de disques sous pression de gaz peuvent être réalisés dans une gamme étendue de température, de 20 à $900{ }^{\circ} \mathrm{C}$ dans cette étude et jusqu'à $-176{ }^{\circ} \mathrm{C}$ dans d'autres laboratoires; bien sûr, la gamme de température est beaucoup moins étendue pour le gonflement hydraulique.

Les plus grandes vitesses de pressurisation donnent des ruptures de disques en une fraction de seconde; les vitesses de déformation des disques atteignent $10^{0} \mathrm{~s}^{-1}$; cette valeur reste très inférieure à la vitesse de déformation atteinte avec des mises en charge par des systèmes de chocs mécaniques ou par des explosifs; en contre partie, les essais de disques à grande vitesse sont facilement réalisés et exploités dans toute la gamme de température jusqu'à $900^{\circ} \mathrm{C}$.

\subsubsection{Sensibilité de l'essai de disques}

Puisque les bords des éprouvettes ne sont pas sollicités pendant l'essai de disques, les défauts de découpe ou d'usinage n'amorcent pas de déformations localisées responsables d'une dispersion de résultats. Comme les résultats sont peu dispersés, ils sont fiables et reproductibles et les phénomènes métallurgiques secondaires ne sont pas masqués ; ainsi, nous avons montré que les essais de disques sont très sensibles à des évolutions microscopiques du matériau comme la précipitation intergranulaire dans le cuivre peu allié.

D'autres études ont montré cette sensibilité à des paramètres microscopiques; pour des alliages de nickel [10], les disques étaient pressurisés jusqu'à leur rupture sans que soient révélées des anomalies de leur comportement mécanique; par contre, le spectromètre de masses 
détectait de très fines fuites d'hélium pour des pressions inférieures à $10 \%$ de la pression de rupture; ces fuites étaient dues à des fissurations traversantes consécutives à des évolutions métallurgiques à haute température. Ces évolutions n'auraient pas été révélées par un essai mécanique classique comme la traction; contrairement au cas de l'alliage de cuivre, les évolutions étaient trop faibles pour modifier le comportement mécanique du matériau.

De plus, la géométrie du disque et de la cellule d'essais donne des courbes expérimentales au domaine de plasticité très dilaté; ainsi, pour le cuivre peu allié, le domaine de plasticité existe entre des pressions dont le rapport est supérieur à 15 (Fig. 3) alors que la charge unitaire intrinsèque du matériau évolue dans un domaine relatif 10 fois plus faible (Fig. 4). Cette dilatation du domaine de plasticité permet de mettre en évidence de faibles différences de comportement entre des matériaux à résistance similaire.

\section{Conclusion}

La méthode de calcul des propriétés mécaniques du disque en traction biaxiale est validée puisqu'elle permet de retrouver les caractéristiques mécaniques déterminées en traction uniaxiale. Par rapport aux essais habituels de traction, l'intérêt de l'essai biaxial de disques est de fournir une caractérisation thermomécanique plus complète des matériaux en feuilles puisque que les gammes de température et de vitesse sont très étendues et que la sensibilité à des mécanismes ou phénomènes secondaires est bien meilleure.

Le calcul analytique proposé permet de déterminer les propriétés mécaniques rationnelles du matériau dans son domaine de plasticité en ne tenant compte que des sollicitations de traction biaxiale du disque; les résultats présentés peuvent être affinés en tenant compte de la flexion dans le disque déformé en coupole; le comportement du matériau peut être complété par le calcul des contraintes et les déformations dans le domaine d'élasticité grâce aux formules établies pour des plaques minces soumises à chargement mécanique réparti.

L'analyse fine des contraintes et des déformations dans les domaines d'élasticité et de plasticité doit permettre de connaitre au mieux le comportement thermomécanique des matériaux dans des gammes larges de température et de vitesse; le comportement pourra alors être modélisé grâce à des lois plus précises que la loi d'Hollomon présentée dans cette étude.

La comparaison des paramètres des lois thermomécaniques obtenues à partir de disques pressurisés par un gaz neutre ou non, permettra une approche rationnelle des modifications dues à l'environnement, comme celles découlant des interactions métal-hydrogène par exemple.

\section{Références}

[1] J.P. Fidelle, R. Broudeur, C. Roux, Les essais de disques sous pression, Ivry, France, Éd. CEA, mai 1975, pp. 7-48
[2] J.P. Fidelle, Present status of the disk pressure test for hydrogen embrittlements, 2nd A.S.M., Symposium on hydrogen embrittlement, Los Angeles, USA, mai 1985, pp. $153-171$

[3] J.P. Fidelle, P. Jouinot, M. Stasi, M. Barthélémy, The range of application of disk pressure test, 5th International conference on hydrogen effects on material behaviour, Jackson Lake, USA, Sep. 1994, pp. 73-78

[4] H. Barthélémy, G. Pressouyre, Hydrogen gas embrittlement of steels, European Economic Community, Report EUR 9730EN, Bruxelles, Belgium, 1985, pp. 14-21

[5] Gas containers, Cylinders and containers for compressed hydrogen, Test method for selecting construction materials, French standard NF E 29-732, Oct. 1990

[6] G. Vibrans, A. Speitling, Investigation of hydrogen induced cracking of tempered 35CrMo4 during disk pressure test, 4th International conference "Hydrogen and materials", Beijing, China, May 1988, pp. 270-278

[7] E.J. Bacheleyt, A.R. Troiano, Hydrogen gas embrittlement and disk pressure test, Report NASA CR 134551, USA

[8] V. Gantchenko, P. Jouinot, M. Stasi, Mechanical properties and failure analysis of metals and plastics cut off from a sheet or a vessel thin wall, EMAS, UK, Milano, Italy, 1999, pp. 391-400

[9] V. Gantchenko, P. Jouinot, M. Stasi, Études de matériaux par des essais de disques sous hélium, une technique sensible et performante, Matériaux et Techniques 87 (1999) 53-60

[10] J. Genevois-Stasi, Étude de l'évolution des propriétés mécaniques et métallurgiques de l'inconel 625 au cours du vieillissement, utilisation de l'essai de disques sous pression de gaz, Thèse, Paris 6, juin 1998

[11] R. Sowerby, J.L. Duncan, Failure in sheet metal in biaxialtension, Int. J. Mech. Sci. 13 (1971) 217-229

[12] R. Hill, A theory of the plastic bulging of a metal diaphragm by lateral pressure, Phil. Mag. 41 (1950) 1133-1142

[13] R. Mesrar, Comportement plastique des tôles sous sollicitation biaxiale et analyse numérique de la mise en forme par gonflement hydraulique, Thèse, Metz, 1991

[14] A. Boualila, M. Ayadi, A. Zghal, K. Jendoubil, Validation expérimentale du modèle de calcul en calotte sphérique de plaques circulaires minces sous l'effet d'un gonflement hydraulique, Mécanique et Industries 3 (2002) 627-638

[15] P. Jouinot, V. Gantchenko, M. Stasi, P. Azou, High pressure disk test aided by computer, 4th International conference "Hydrogen and materials", Beijing, China, mai 1988, pp. 278-287

[16] P. Jouinot, Développement de l'essai de disques sous pression, Applications à la fragilisation par l'hydrogène d'aciers faiblement alliés, Thèse, Paris 6, Déc. 1991

[17] D. François, Essais mécaniques des matériaux, Détermination des lois de comportement, Techniques de l'ingénieur M120

[18] F. Saint-Antonin, Essais de fluage, Techniques de l'ingénieur M140

[19] P. Rabbe, H.P. Lieurade, A. Galtier, Les essais de fatigue, Techniques de l'ingénieur M4171

[20] S. Timoshenko, S. Woinowsky-Krieger, Theory of Plates and Shells, Mc Graw-Hill Book, Company, Inc., 1959, pp. $51-98$ 